\title{
NOTA TAXONOMICA SOBRE IBERIS FONTQUERI PAU, ENDEMISMO MALAGUEÑO
}

\author{
M. MORENO
}

RESUMEN: Se estudian aspectos morfológicos, anatómicos, biométricos y citológicos de Iberis fontqueri Pau, mostrando su originalidad entre las especies del género.

SUMAARY: Morphological, anatomical, biometrical and citologycal features of Iberis fontqueri Pau are studied. The originality of this species about the others of the genus is demostrated.

\section{INTRODUCCION}

Este taxon fué descrito por Pau (1922) a partir de los ejemplares herborizados por Gros en 1919. Muy escasas deben ser las recolecciones anteriores a esta fecha pues, entre los numerosos herbarios nacionales y europeos consultados, solamente hemos encontrado un pliego de Willkomm, fechado en 1845, quien la denomina I. pinnata L. al igual que Boissier (1839). Tampoco las recolecciones posteriores son muy abundantes.

En el marco de un estudio más global de las especies endémicas del género Iberis L. en la Península Ibérica, I. fontqueri Pau destacó enseguida por su singularidad, tanto en los aspectos morfológicos, como en los elementos epidérmicos, semilla, número cromosómico, etc., como veremos en los apartados siguientes.

\section{MATERIAL Y METODOS} de :

Los especímenes de I. fontqueri que fueron analizados proceden

MALAGA: Sierra Romera (Estepona) y Barranco del Madroñal y Minas (Sierra de Yunquera) 16-18.V.1919, Gros (Tipo, MA 44824) -taxonomía numérica-; Sierra Palmitera (nudo de Ronda) 23.V.1972, Sañudo \& Fernández Casa (SEV 10876) -taxonomía numérica-; Sierra Bermeja, 4.V.1931, Vicioso (MA 44826) -taxonomía numérica-; Ibídem, 1.VI.1966, Borja y Rivas Goday (MA 186990) -taxonomía numérica-; Ibídem, 25.V.1966, sin rec. (SEV sin número) -taxonomía numérica, epidermis foliar-; Ibídem, 19.V.1979, Rivas Goday e Izco (MAF 82120) -biometría polínica-; Ibídem, 18.IV.1845, Willkomm (COI herb. Willk.) -taxonomía numérica-; Ibídem, 16.VIII.1978, Molesworth Allen -bio- 
metría seminal, cariología-; Ibídem, subida desde Estepona al Puerto de Peñas Blancas, 16.V.1980, Alsina, Fernández, Mendoza y Moreno -taxonomía numérica-; Ibídem, carretera Jubrique-Estepona, 15.V.1980, Alsina, Fernández, Mendoza y Moreno -taxonomía numérica, biometría polínica, exina al MEB, epidermis foliar-, Ibídem, 19.VIII.1981, Mendoza y Moreno - testa se minal al MEB, cariología-.

No incluimos aquí el material utilizado para otras especies de Iberis (Moreno, 1984) para no hacer la lista excesivamente larga.

En el estudio morfológico se aplicaron las técnicas de taxonomía numérica (Sokal \& Sneath, 1973) comenzando por elaborar una matriz de datos con 156 caracteres analizados para un total de 96 unidades taxonómicas operativas (UTO), de las cuales, 8 eran de I. fontqueri. Esta matriz se procesó mediante un programa de análisis factorial de correspondencias (Ornduff \& Crovello, 1968; Mc Neill, 1979).

El material polínico fue acetolizado (Erdtman 1952) y posteriormente medido en sus ejes polares - $\mathrm{P}-\mathrm{y}$ en sus diámetros ecuatoriales -E-, permitiendo la comparación de los resultados.

El mismo tratamiento biométrico recibieron las semillas y los elementos -células y estomas- de la epidermis foliar; estos últimos se obtuvieron tras ablandar las hojas en solución de potasa al 1:1000, raśpado y decoloración mediante hipoclorito sódico (Bellot, 1943).

Realizamos recuentos cromosómicos en células de meristemo radicular teñidas con orceina acética y posteriormente aplastadas(La Cour, 1954).

\section{RESULTADOS}

\section{Morfología}

En la representación cartesiana de la matriz de datos original, sobre los planos definidos por los ejes 1 y 3 (Gráfica 1), podemos apreciar los siguientes hechos:

La total independencia del conjunto de elementos de I. fontqueri con respecto a los de las otras especies.

El elevado grado de homogeneidad intraespecífica en el seno de I. fontqueri, lo que se evidencia en que el ordenador sólo ha podido escribir 5 de las 8 UTO introducidas ya que las tres restantes se superpondrían con alguna otra.

Desde el punto de vista morfológico, I. fontqueri Pau es una planta bastante singular, pudiendo destacar que: especies.

Es enteramente glabra, carácter que comparte con muy pocas

Sus hojas basales, especialmente cuando jóvenes, presentan tonos morado-negruzcos mucho más llamativos que los tintes purpúreos que hemos observado en algunos ejemplares de I. nazarita o de I. grossi.

La forma de sus frutos es inconfudible y claramente distinta a la de cualqiuer otro Iberis.

Sus estigmas son apenas emarginados, incluso en los estadios más maduros, lo que no es frecuente en el género. co.

Lo más notable, sin embargo, es su escaso dimorfismo petáli- 


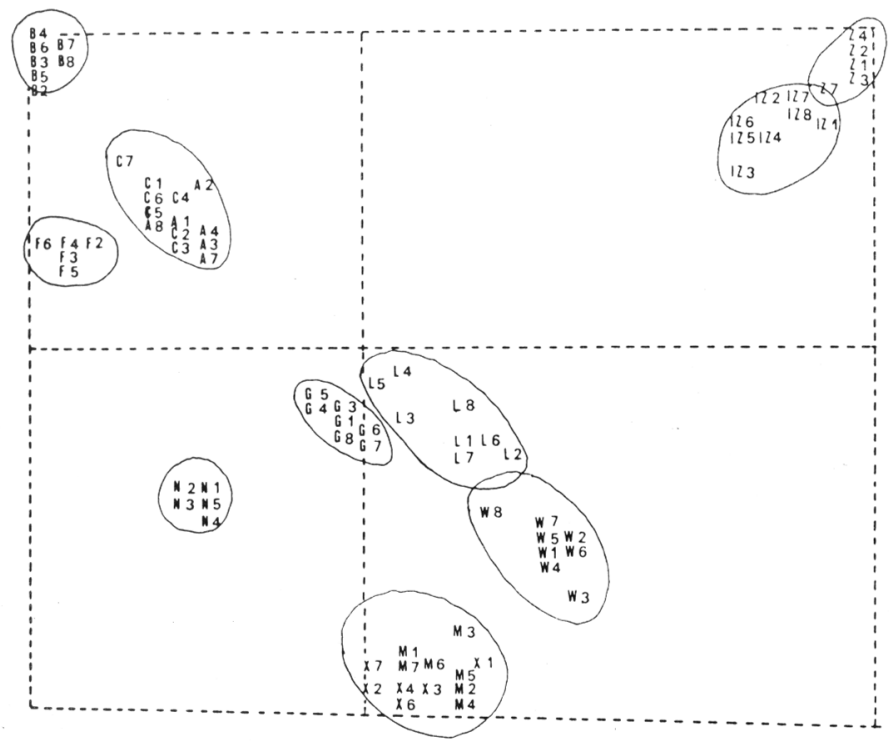

Gráfica 1.- Resultado del análisis factorial de correspondencias. $F=$ UTO de I. fontqueri Pau; $C=I$. crenata Lam.; $A=I$. sampaiana Amaral Franco E Pinto da Silva; $G=I$. grosii Pau; $B=I$. gibraltarica L.; $N=I$. nazarita Moreno; $Z$ = I. saxatilis L. subsp. saxatilis; $I Z=I$. saxatilis L. subsp. cinerea (Poir.) Font Quer; $L=I$. linifolia Löefl. subsp. linifolia; $W=$ I. linifolia Löefl. subps. welwitschii (Boiss.) Amaral Franco \& Pinto da Silva; $X$ = I. procumbens Lge. subsp. procumbens; $M=I$. procumbens Lge. subsp. microcarpa Amaral Franco \& Pinto da Silva.

\begin{tabular}{|c|c|c|c|c|c|c|c|c|}
\hline \multirow{3}{*}{ Datos en micras } & \multicolumn{4}{|c|}{ Haz hojas medianas } & \multicolumn{4}{|c|}{ Envés hojas medianas } \\
\hline & \multicolumn{2}{|c|}{ Céls. epidér. } & \multicolumn{2}{|c|}{ Estomas } & \multicolumn{2}{|c|}{ Céls. epidér. } & \multicolumn{2}{|c|}{ Estomas } \\
\hline & $\bar{L}$ & $\bar{A}$ & $\bar{L}$ & $\bar{A}$ & $\bar{L}$ & $\bar{A}$ & $\bar{L}$ & $\bar{A}$ \\
\hline I. bernardiana & 89,9 & 50,7 & 24,9 & 21,6 & 95,8 & 62,3 & 25,6 & 22,2 \\
\hline I. crenata & 120,2 & 63,3 & 24,9 & 21,2 & 119,3 & 63,0 & 24,5 & 20,8 \\
\hline I. fontqueri & 150,1 & 85,0 & 30,5 & 24,0 & 173,7 & 92,6 & 30,1 & 24,2 \\
\hline I. nazarita & 103,1 & 65,0 & 25,2 & 23,1 & 99,4 & 60,8 & 26,8 & 24,9 \\
\hline I. gibraltarica & 77,1 & 39,1 & 23,5 & 20,2 & 79,3 & $35,7^{\circ}$ & 23,9 & 19,1 \\
\hline I. grosii & 82,3 & 47,4 & 24,3 & 19,6 & 103,9 & 62,9 & 35,2 & 20,2 \\
\hline I. procumbens & 114,1 & 62,1 & 28,1 & 24,6 & 113,9 & 58,0 & 29,0 & 24,7 \\
\hline I. saxatilis & 88,3 & 44,9 & 27,4 & 22,1 & 87,8 & 45,6 & 27,2 & 22,6 \\
\hline I. spathulata & 77,4 & 62,7 & 22,1 & 20,7 & 92,3 & 49,9 & 24,5 & 23,8 \\
\hline I. linifolia & 90,9 & 49,0 & 27,2 & 23,1 & 104,3 & 61,7 & 23,9 & 23,8 \\
\hline I. pruitii & 87,2 & 54,5 & 26,4 & 22,3 & 114,4 & 60,0 & 27,3 & 22,5 \\
\hline
\end{tabular}




\section{Epidermis foliar}

Las células epidérmicas de I. fontqueri (Lám. 1, fig. 8) son muy irregulares, oblongas y de contornos sinuosos; es sin duda la especie de Iberis que presenta estos elementos de mayor tamaño, con longitudes comprendidas entre 150-170 umaproximadamente (Cuadro 1).

Sus estomas aparecen distribuídos sin orden aparente en toda la superficie foliar, tanto en haz como en envés, y son de morfología oblonga y tamaño considerable.

\section{Polen}

El grano de polen de I. fontqueri (Lam. 1, fig. 1, 2, 3 y 4) con dimensiones de $\overline{\mathrm{P}}=28,3 \mu \mathrm{my} \bar{E}=24,9 \mu \mathrm{m}$ es de los más grandes entre los estudiados (Cuadro 2) únicamente superado por el I. gibraltarica e I. nazarita.

\section{Semilla}

I. fontqueri presentó el menor de los tamaños seminales de entre todas las especies estudiadas (Cuadro 3 ).

Su testa seminal, vista con el MEB (Lám. 1, fig. 9) mostró una ornamentación de aspecto cerebroide, coincidiendo únicamente con I. bernardiana, claramente diferente de los modelos más o menos estriados, foveolados reticulados, etc. de las demás especies.

\section{Citología}

El número cromosómico de I. fontqueri Pau resultó ser $2 \mathrm{n}=16$, con lo que nuevamente esta especie se muestra original ya que, salvo I. sempervirens L. var. pseudosaxatilis (Emberger) Maire mencionado por Quezel (1957) y discutido por Favarger, Galland \& Küpper (1980), quienes obtienen para esa misma planta $2 \mathrm{n}=22$, no tenemos noticia de ninguna otra especie del género con genoma $\mathrm{n}=8$.

Iberis fontqueri Pau, Mem. Mus. Ci. Nat. Barcelona, Ser. Bot., 1(1): 22. (1922). (Láminas 2 y 3)

Biótipo terófito, careciendo de roseta foliar estéril. Ramificación de un eje solitario ramificado o no en su parte alta, o numerosos saliendo desde la base (nuevamente ramificados o no) alcanzando o sobrepasando al principal, todos acabados en corimbos floridos (Lám. 2). Tallos de 10-20 cm, herbáceos, con estrías superficiales poco marcadas, enteramente glabros. Hojas basales oblongoespatuladas, anchas, atenuadas en largo peciolo, con dos o tres lóbulos o anchos dientes obtusos en su mitad distal, de 15-17 × 5-7 mm, presentan un característico color púrpura oscuro; las superiores más estrechas, linearespatuladas a lineares, enteras o con un par de dientes, de 10-15 x 1$2 \mathrm{~mm}$, todas glabras (Lám. 2). Epidermis foliar con células enormes de contornos irregulares, midiendo 150-170 x 80-90 $\mu$ y estomas muy oblongos de $30 \times 24$ н. Inflorescencia e infrutescencia (Lám. 2) en corimbos de pequeñas dimensiones, bastante planos, no muy densos y contraidos en fruto. Botón floral muy alargado y minúsculo (Lám. 3, a y b). Flor de sépalos ovaloblongos, bordes enteros, glabros, de $2,5 \times 1 \mathrm{~mm}$, erectos en la flor joven y patentes en la madurez, teñidos de púrpura en su parte superior, todos, incluso los medianos, 


\begin{tabular}{|l|c|c|}
\hline \multicolumn{1}{|c|}{ Datos en micras } & $\overline{\mathrm{P}}$ & $\overline{\mathrm{E}}$ \\
\hline I. bernardiana & 25,8 & 21,6 \\
I. crenata & 24,7 & 22,7 \\
I. fontqueri & 28,3 & 24,9 \\
I. nazarita & 28,8 & 26,2 \\
I. gibraltarica & 30,0 & 25,7 \\
I. grosii & 26,2 & 22,7 \\
I. procumbens & 25,9 & 23,8 \\
I. saxatilis & 25,7 & 23,6 \\
I. spathulata & 27,2 & 24,6 \\
I. linifolia & 26,7 & 23,9 \\
I. pruitii & 26,9 & 24,3 \\
\hline
\end{tabular}

Cuadro 2.- Valores medios en micras, de los ejes polinicos en los táxones estudiados.

\begin{tabular}{|l|c|c|c|}
\hline \multicolumn{1}{|c|}{ Datos en milímetros } & long. & anch. & esp. \\
\hline I. bernardiana & 1,97 & 1,37 & 0,59 \\
I. crenata & 2,34 & 1,49 & 0,73 \\
I. fontqueri & 1,74 & 1,13 & 0,47 \\
I. nazarita & 2,70 & 1,56 & 0,58 \\
I. gibraltarica & 5,00 & 3,68 & - \\
I. grosii & 2,55 & 1,61 & 0,74 \\
I. procumbens & 2,51 & 1,57 & 0,61 \\
I. saxatilis & 3,08 & 2,26 & 0,78 \\
I. spathulata & 2,66 & 1,65 & 0,55 \\
I. linifolia & 2,48 & 1,49 & 0,59 \\
I. pruitii & 2,64 & 1,63 & 0,72 \\
\hline
\end{tabular}

Cuadro 3.- Valores medios en $\mathrm{mm}$, de longitud, anchura y espesor seminales. 
sacciformes en la base, no persistentes (Lám. 3, $c_{1}$ y $c_{2}$ ); pétalos ovalespatulados, muy estrechos, de $7 \times 2 \mathrm{~mm}$, escaso dimorfismo petálico, blancos con venas en ocasiones violeta (Lám. 3, d). Estambres de $4 \mathrm{~mm}$, antera de c. $1 \mathrm{~mm}$ (Lám. 3, e). Polen de $28 \times 25 \mu \mathrm{m}$ en el límite entre esferoidal y subprolato cuando está acetolizado, exina ornamentada con un fino retículo. Pistilo de $3,5 \mathrm{~mm}$, lóbulos superiores inconspícuos. Estilo de $2 \mathrm{~mm}$, prismático (Lám. 3, f) y estigma capitado, poco o nada emarginado en la madurez, papiloso en toda su superficie (Lám. 3, g). Nectarios no muy grandes (Lám. 3, f). Pedicelos fructíferos con costillas poco marcadas, planos en su lado adaxial (Lám. 3, h), muy cortos. Fruto de cuadrado a oboval, más ancho en su parte superior, de $6 \times 5 \mathrm{~mm}$, con amplios y cortos lóbulos superiores de borde aserrado, escotadura muy obtusa y superficial, estilo muy exerto, replo uniforme de c. $1 \mathrm{~mm}$ (Lám. 3, i). Semilla ovalada, carente de ala, midiendo $1,7 \times 1,1 \times 0,5 \mathrm{~mm}$ (Lám. 3, j), color amarillo oscuro o marrón claro, testa de aspecto cerebroide.

Tipo: (MA; lectótipo). El pliego MA 44824 "I. fontqueri Pau n. sp. Sierra de la Romera (Estepona) y Barranco del Madroñal y Minas (Sierra Yunquera). 16-18.V.1919, E. Gros" contiene cinco pies de planta muy deteriorados; elegimos como lectótipo al ejemplar de la parte inferior izquierda. Agosto.

Fenología: Florece en Mayo y maduran los frutos en Julio y

Hábitat: Sustratos básicos, con frecuencia serpentinas; aparece en taludes y laderas pedregosas entre los 700 y $1.100 \mathrm{~m}$.

Distribución geográfica: Endemismo local de las sierras malagueñas occidentales.

Localidades estudiadas:

MALAGA: Sierra Bermeja, 25.V.1966, sin rec.(SEV sin número); Ibídem, 1.VI.1966, Rivas Goday, Borja, Ladero y Mayor (SAL 8462, MAF 10310, MAF 94136, MAF 68383, MAF 186990); Ibídem, 28.V.1926, Gros (BC 90311); Ibídem, 18.IV.1845, Willkomm (COI herb. Willk. sin número); Ibídem, 19.V.1969, Rivas Goday e Izco (MAF 82120); Mijas, 4.V.1931, Vicioso (MA 44826); Estepona, 9.VI.1969, Asensi y Diez (MGC sin número, MAF 105486); Carretera Jubrique-Estepona, 15.V.1980, Alsina, Fernández, Mendoza y Moreno; Subiendo desde Estepona al Puerto de Peñas Blancas, 16.V.1980, Alsina, Fernández, Mendoza y Moreno; Sierra Palmitera, 11.VI.1976, Mazimpaka, Pajarón y Ron (MAC 4287); Ibídem, nudo de Ronda, bajando a San Pedro, 23.v.1972, Sañudo y Fernández Casas (SAL 4004, SEV 10876); Sierra de la Romera, prope Estepona, 16.V.1919, Gros (BC 05174); Ibídem y Barranco del Madroñal y Minas (Sierra Yunquera), 16-18.V.1919, Gros (MA 44824, BC 05172, BC 05173); Sierra de Tolox, cerca de Puerto Corona, 5.VII.1936, Laza (BCF 4618, LISE $19773)$.

Sintaxonomía: En comunidades de Andryaletalia ragusinae y de Phagnalo-Rumicetalia indurati (Rivas Goday \& Esteve, 1972). 


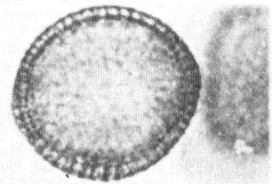

\section{1}

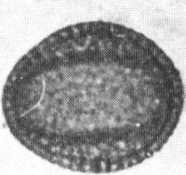

3

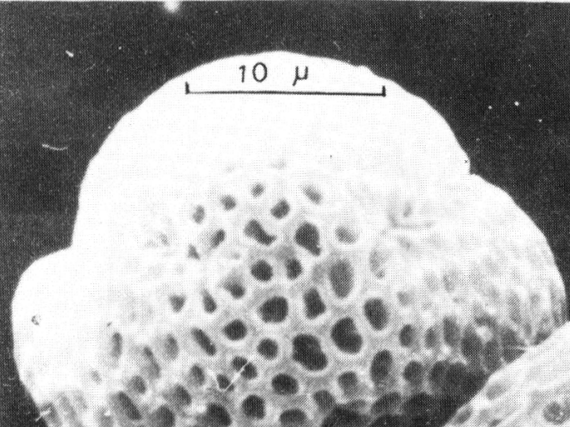

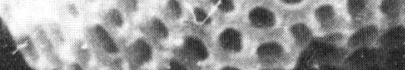

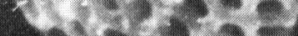

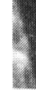

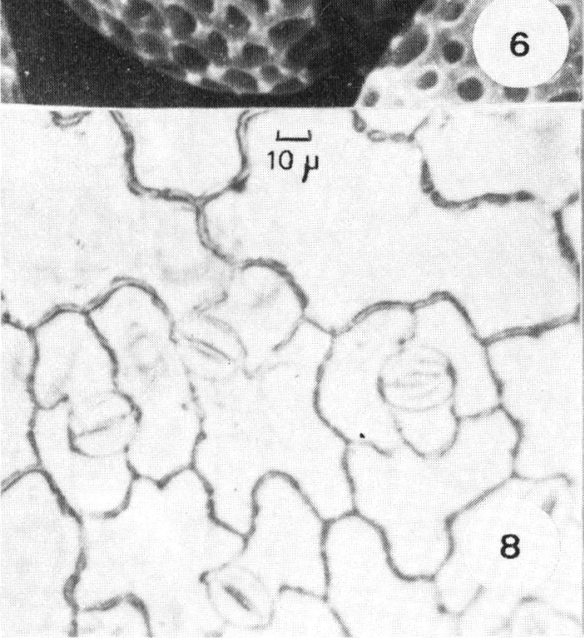

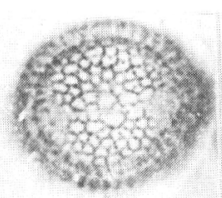

2

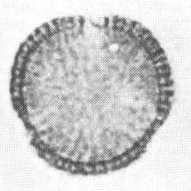

4
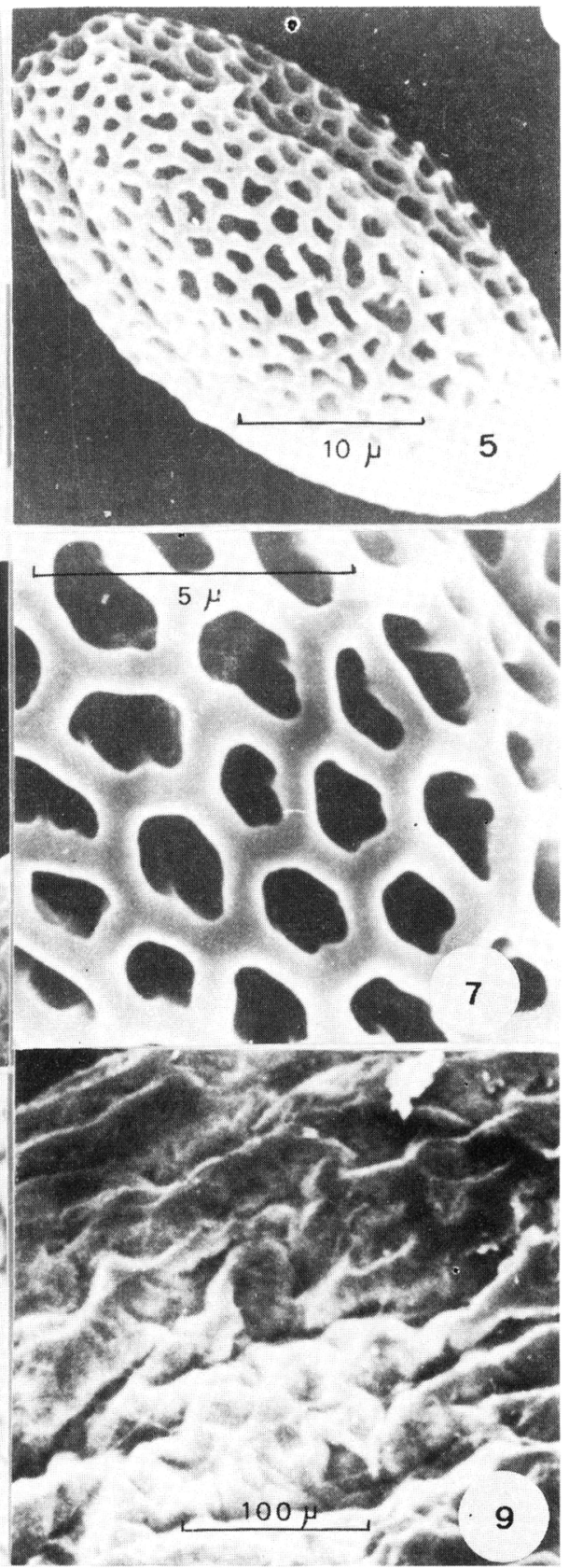

Lámina 1.- Figura 1. grano de polen acetolizado en corte óptico meridiano; Figura 2. ídem, vista superficial; Figura 3. Idem, mostrando dos colpos; Figura 4. idem, vista polar; Figura 5. grano de polen al MEB en vista ecuatorial; Figura 6. idem, en vista polar; Figura 7. detalle de la exina: reticulo de la zona interapertural; Figura 8 . epidermis foliar del haz; Figura 9. Detalle de la superficie seminal al MEB. 


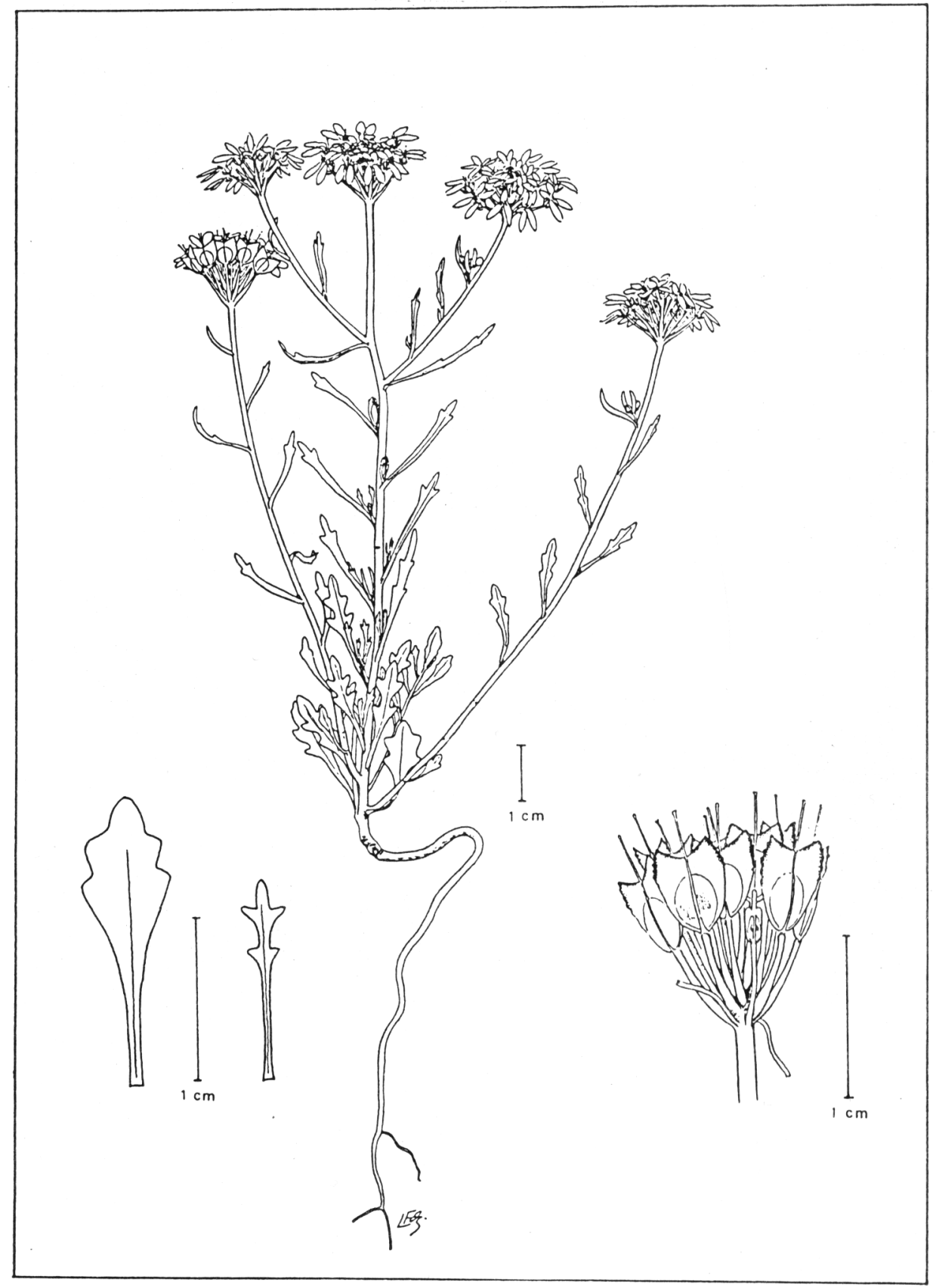

Lámina 2. Aspecto general y detalles de I. fontqueri Pau. Ejemplar de Málaga: Sierra Bermeja, Jubrique. 


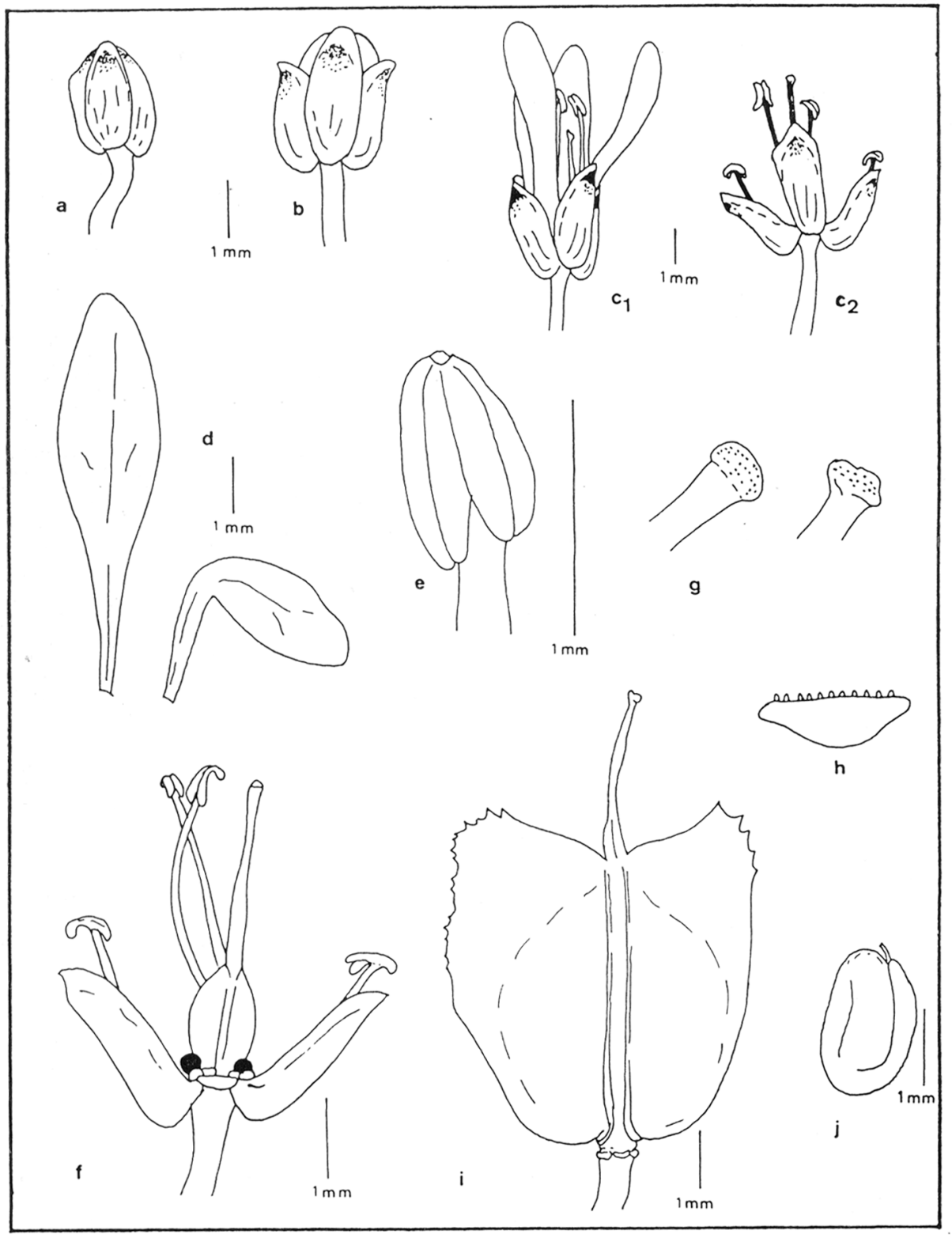

Lámina 3. Detalles anatómicos de I. fontqueri Pau. a) botón floral cerrado; b) ídem, abierto; $\left.c_{1}\right)$ flor recien desplegada mostrando los sépalos erectos; $c_{2}$ ) flor madura mostrando los sépalos patentes; d) pétalos; e) antera; f) gineceo y nectarios; g) estigmas; h) sección de un pedicelo fructífero; i) fruto; j) semilla. 


\section{B I BL IOGRA F I A}

BELLOT, F. -1943- Contribución al estudio de la orientación de los estomas en las epidermis vegetales. An. Inst. J.C. Mutis de Farmacognosia, 2(3) : 137-212.

BOISSIER, E. -1839- Voyage Botanique dans le Midi de l'Espagne pendant 1 'année 1837. 2: 54-55. París.

ERDTMAN, G. -1952- Pollen morphology and Plant taxonomy. Almquist and Wiksell. Stockholm.

FAVARGER, S., GALLAND, N. \& KUPFER, PH. -1980- Recherches cytotaxonomiques sur la flore orophile du Maroc. Naturalia Monspeliensia, 29: 2930 .

LA COUR, F. -1954- Smear and squaser tecniques in plant cytology. Labor Practice, 3: 326-330.

MC NEILL, J. -1979- Structural value: a concept used in the construction of taxonomic classifications. Taxon, 28(3/6):481-504.

MORENO, M. -1984- Taxonomía de las especies endémicas del género Iberis L. (Cruciferas) en la Península Ibérica. Univ. Compl. Madrid.

ORNDUFF, R. \& CROVELLO, T.J. -1968- Numerical taxonomy of Limnantaceae. Amer. J. Bot., 55(2): 173-182.

PAU, C. -1922- Nueva contribución al estudio de la flora de Granada. Mem. Mus. Ci. Nat. Barcelona, ser. Bot., 1(1): 21-22.

QUEZEL, P. -1957- Peuplement végétal des hautes montagnes de l'Afrique du Nord. Encycl. Biogéograph. et Ecol., 10.137: 1-406.

RIVAS GODAY, S. \& ESTEVE, F. -1972- Flora serpentinícola española. Nota segunda. Anal. Real. Acad. Farm., 38: 409-462.

SOKAL, R.R. \& SNEATH, P.H. -1973- Numerical taxonomy, the principles and practice of numerical classification. Freeman. San Francisco. 